\title{
THE EFFECT OF A PRELIMINARY HAUSMAN TEST ON CONFIDENCE INTERVALS
}

\author{
RHEANNA MAINZER
}

(Received 4 June 2018; first published online 15 August 2018)

2010 Mathematics subject classification: primary 62F25; secondary 62J05.

Keywords and phrases: coverage probability, confidence interval, Hausman specification test, model selection.

The Hausman pretest [1] is frequently used to decide if the time-varying covariate is exogenous in a random effects model for panel data. Commonly in practice, if the null hypothesis that the covariate is exogenous is accepted then the random effects model is used for subsequent inference; otherwise the fixed effects model is used. In a detailed analysis we show that a confidence interval for a slope parameter constructed after this pretest has poor coverage and expected length properties in comparison to the confidence interval constructed using the fixed effects model. We then modify this post-model-selection interval so that it has minimum coverage probability that is (to an excellent approximation) equal to the nominal coverage and favourable expected length properties. Part of this work appears in [3, 4].

Following this, we investigate why post-model-selection confidence intervals can have such poor coverage properties. Is it due to the fact that we use the same data twice: once to choose the model and a second time to construct a confidence interval? Or is it that the preliminary model selection sometimes chooses the wrong model? This work appears in [2]. Lastly, we build on the work of Turek and Fletcher [5], who proposed a model averaged tail area (MATA) confidence interval. We optimise the weight function for this confidence interval in the scenario of two nested linear regression models. This results in a family of MATA intervals with ideal coverage and improved expected length properties, in the context of this scenario.

Thesis submitted to La Trobe University in November 2017; degree awarded on 16 April 2018; supervisors Paul Kabaila and Davide Farchione.

(C) 2018 Australian Mathematical Publishing Association Inc. 


\section{References}

[1] J. A. Hausman, 'Specification tests in econometrics', Econometrica 46 (1978), 1251-1271.

[2] P. Kabaila and R. Mainzer, 'Two sources of poor coverage of confidence intervals after model selection', Statist. Probab. Lett. 140 (2018), 185-190.

[3] P. Kabaila, R. Mainzer and D. Farchione, 'The impact of a Hausman pretest, applied to panel data, on the coverage probability of confidence intervals', Econom. Lett. 131 (2015), 12-15.

[4] P. Kabaila, R. Mainzer and D. Farchione, 'Conditional assessment of the impact of a Hausman pretest on confidence intervals', Stat. Neerl. 71 (2017), 240-262.

[5] D. Turek and D. Fletcher, 'Model-averaged Wald intervals', Comput. Statist. Data Anal. 56 (2012), 2809-2815.

RHEANNA MAINZER, School of Mathematics and Statistics, University of Melbourne, Parkville, VIC 3010, Australia

e-mail: rheanna.mainzer@unimelb.edu.au 